\title{
The Characteristics of Wang Wei 's Paintings in Tang Dynasty and Their Teaching Enlightenment
}

\author{
Jing Wang \\ Xi'an University School of Humanities, Xi'an University, 710065
}

Keywords: Wang wei's painting; Ink landscapes; North and south sect; Teaching

\begin{abstract}
As the originator of the literati painting, Wang Wei is similar to Wu Daozi, Li Sixun and Li Zhaodao in terms of painting. However, Wang Wei is unique in the subject matter and technique. Based on the times he lived in, this article analyzes Wang Wei's painting theme, style characteristics and the status of the Chinese painting and attempts to draw a true outline of Wang Wei, which will have some enlightenment on art history and related teaching.
\end{abstract}

\section{Introduction}

Wang Wei's painting is generally recognized as Wang Wei pioneered the first of literati painting. Besides, Wang Wei in the painting even exceeded his status as a poet. Starting from the general cognition of Wang Wei, this article attempts to restore the image of Wang Wei who is a real painter to have a correct understanding and cognition in teaching.

\section{Theme of Wang Wei's Paintings}

The most famous painting of Wang Wei is "landscape painting", but he is not only painting landscapes. Wang Wei's paintings has been lost due to the passage of time. His most famous painting "Wang Chuan Tu" makes people easily misunderstand Wang Wei as a pure landscape painter.

Wang Wei Paints Both Characters and Landscape. Wang Wei's paintings spread more widely are as follows, such as "Wang Chuan Tu", "Xiangyang Meng Gong Read Poetry on Horse", "Jiang Shan Xue Ji Tu" and "Fu Sheng Shou Jing Tu". There are 126 Wang Wei paintings recorded in Xuan He Paintings, including 72 figure paintings, 1 animal portrait and 53 landscapes. Digitally speaking, the number of figure paintings actually surpassed the landscape painting, breaking Wang Wei's impression famous for his landscape. The figure painting is divided into two kinds, one is depicting people in real life and the other is depicting the imaginary Buddhist idol. Among these paintings, there are paintings on silk and murals.

"Quan Tang Shi" records that Ouyang Jiong portrays in the poem "Shan Yue Da Shi Ge" about the arhats drawn by Guan Xiu from late Tang Dynasty to the Five Dynasties. At the same time, it is compared with Wu Daozi and Wang Wei of the same dynasty to highlight the superb artistry of Guan Xiu. Wu Daozi is the leading painter in the Tang Dynasty. With Wang Wei and Wu Daozi set off Guan Xiu that praised in poems, it can also reflect the Wang Wei's art of painting recognition from one aspect. Of course, we should compare Wang Wei's figure painting or Buddha images with Guan Xiu's, otherwise there is no comparability. Therefore, Wang Wei's figure painting are at least outstanding in the eyes of Ouyang Jiong who is a poet in the late Tang Dynasty. In addition, "History of Chinese Painting Chronicles" also mentioned that "in addition to landscapes, Wang Wei also good at painting people." According to legend, we can indeed see his character changes in the color of the line, the flexible curve and the texture of the skin from the works of Wang Wei "Fu Sheng Shou Jing Tu", which also reflects the strong realistic ability. In this picture, Lohan looks like an ordinary man, which shows his characterization of portraits: that is, he attaches great importance to reflect the authenticity of objective life.

Therefore, although Wang Wei is famous for his landscape, Wang Wei's figure painting is still considerable and of considerable standard in the Tang Dynasty, where portraits were the most important paintings. 
Most of Themes of Wang Wei's Paintings Are Landscapes and It Has Become His Characteristic. The figure painting is the highest achievement in Tang dynasty. Experienced the long-term development and absorbed the essence from Qin Dynasty to Jin Dynasty, Wu Daozi set a paradigm for figure painting, leading the figure painting in the Tang Dynasty into a superb and mature stage. Therefore, painters are mainly figure painter and they are the most famous in Tang Dynasty.

In Wang Wei's paintings, nearly half of his paintings are landscape paintings according to "Xuan He Paintings". Comparatively speaking, "Xuan He Paintings" records Wang Wei's landscape paintings in more detail with different names. There are pictures of the snow-capped mountains, the journey of the valley, the villas, the snow scene to be crossed, the mountains and the countryside, the morning and many others, showing Wang Wei's extensive themes in landscape painting. Painters who draw snow in the same period are rare.

"Xuan He Painting" evaluates that Wang Wei is good at painting and landscapes, and he masters very comprehensive techniques. "Old Book of Tang" also mentioned Wang Wei's exquisite painting and calligraphy, and his landscape painting is good at depicting the real statues. "Tang Dynasty History" also mentioned Wang Wei's paintings technique is extremely high, especially the far-reaching landscape in some housing murals. "Tang Dynasty Famous Paintings" also refers to the landscape of Wang Wei. Turquoise is similar to Wu Daozi's painting, and the Qing Feng's drawings preserving in Qianfu Temple have their own characteristics. When referring to Wang Wei's paintings, there are criticisms about the characters, but more are still landscapes and the evaluation is even higher. Gao Juhan also mentioned Wang Wei's contribution to the development of ink painting more than his figure paintings.

It can be seen that Wang Wei's painting still has its focus on the basis of a comprehensive study and the number of figure paintings is large. However, the theme of landscape painting is more and more novel. Judging from the present and future generations, his landscape painting is more representative of its characteristics.

\section{Wang Wei and Ink Landscape}

Wang Wei is known as ink landscapes, but the ink landscape is not from Wang Wei. Wang Wei has its own unique contribution to the ink landscape, breaking the monotonous technique of thick ink coloration and seeking change in the ink itself.

We can see from Wang Wei's painting "Xue Xi Tu" that Wang Wei's snow landscapes has no coloring and only the shades of different colors, which shows the vast expanse of cold snow and gives people the feeling of chill. In the mainstream of green landscape at that time, it should be said that is a great breakthrough.

Ink Landscape Is Not from Wang Wei. According to records, Liu Dan in Tang Dynasty(about 771) is also ink landscape painter. The poet Du Fu used "smoke" and "vitality" in the poem titled "Feng Xian Liu Shao Fu Xin Hua Shan Shui Zhang Ge" in Liu Dan's book to describe the wetness of his paintings.

Zhang Zao, a contemporary artist, is also a painter of ink painting landscape. Zhu Jingxuan said that his landscape painting has deep and cold meaning. Jing Hao also wrote in the "Writing Method of the Scriptures" that Zhang Zao's tree and stone ink is full of charm and without color, which is a great breakthrough.

Wu Daozi, Liu Dan and Zhang Zao are all great painters of the Tang Dynasty. Some of them focus on the themes of Buddhism and some of them consist of landscapes, but they all have ink works and even famous for ink paintings. Therefore, in addition to Wang Wei in the Tang Dynasty, there are still many painters are good at painting ink landscape painting. Even though the years of birth and death were not before Wang Wei and there was no clear basis to show that they were affected by Wang Wei, therefore it could not say that the ink painting started from Wang Wei as he is the earliest ancestors of the South Sect.

Wang Wei's Ink Technique. Jing Hao compared Wang Wei, Li Sixun and Wu Taozi in "Writing Method of the Scriptures" and said that Li Sixun's handwriting is exquisite and subtle and the image 
of Wu Daozi's works is better than good fortune and superb style, but both of their painting lack of ink. Jing Hao is the first one to confirm the achievements of Wang Wei's painting and is also the first to list Wang Wei's position on Wu Daozi and Li Sixun.

Speaking of Wang Wei's ink painting landscape, Zhang Yanyuan described as "desisive" and fiffer from today's understanding of the ink, which refers to the method of breaking the former thick ink flat coating, so that the ink is deep and shallow and highlight the level of painting. Since then, there have been offspring of ink and color and a variety of texturing methods.

Gao Juhan also thinks that Wang Wei's contribution to the history of ink landscape is more than his portrait painting. However, he did not agree with the statement that PoMo and texturing methods started from Wang Wei.

At the early Tang Dynasty landscape painting's techniques are not rich. Zhang Yanyuan is the earliest one to record how to use pen and ink in Tang Dynasty. In his "Famous Paintings in Ancient History", there are two places about PoMo technique. According to the records, Wang Wei born at 701 and died at 761 years. There was no exact birthday of Zhang Zao and only recorded that he painted in in Chang'an at Jianzhong three years (782) and affected by Wang Wei ink painting. Zhang Zao was born late than Wang Wei, so his PoMo technique may be self-created. In time to conclude, it is unlikely that Wang Wei Zhang learn from Zhang Zao's PoMo technique. By the impact of Wang Wei ink painting, his paintings may also contains PoMo technique. Thus it can be seen that the PoMo technique originated from Wang Wei and Zhang Zao in Tang Dynasty. As for whether the two created their own techniques or learned from each other, they could not be conclusive. However, Wang Wei born earlier than Zhang Zao, so it is reasonable that descendants attribute PoMo technique to Wang Wei. Unfortunately, there is no record of texturing methods in Tang Dynasty.

Wang Wei is famous for ink landscape and has made some breakthroughs in ink techniques. Although it still has questions for PoMo technique created by Wang Wei, it is reasonable to follow from the historical records and chronology. While, Cun technique lack of sufficient basis.

\section{Known as the Ancestor of South Sect, Differences between Wang Wei and North Sect on Painting Style or Concept}

To the Ming Dynasty, Wang Wei was respected for the ancestors of the South Sect literati painting by Dong Qichang. And Dong Qichang said that the use of ink is different from green landscape painting, leading the world to follow. However, in the Tang Dynasty, Wang Wei was not as prominent as Dong Qichang said.

Dong Qichang in Ming Dynasty Proposed the Distinction between Northern and Southern Sect Paintings. Dong Qichang, a painting theorists in the Ming Dynasty, puts forward in the "Picture Purposes" that paintings are divided into two parts: North Sect and South Sect. North Sect's representative are Li Sixun and his son with coloring landscapes and the successors are Zhao Gan, Ma Yuan, Xia Gui and others in the Song Dynasty. South Sect starts from Wang Wei with rendering to change the traditional way of coloring. Successors are Zhang Zao, Jing Hao, Guan Tong, Dong Yuan, Ju Ran, Mi Fu, Mi Youren, Yuan Sijia and so on.

Dong Qichang divides landscape painting into two schools based on the style of landscape painting of the north and south of Zen. Northern style of painting is green landscape and the representative are Li Sixun and Li Zhaodao. The representative of South Sect is Wang Wei and his style is ink landscape with shading. Painters are divided into two groups in Song, Yuan and Ming Dynasties. One school mainly paints courtyard paintings and the other school paints literati paintings.

There may be two reasons for dividing Sect from the Tang Dynasty. The one reason is that Buddhism has the north and south of Zen. While Buddhism flourished in Tang Dynasty and also divided the Northern and Southern religions from the Tang Dynasty. The other reason is that landscape paintings came into fruition at the beginning of the Tang Dynasty with a more mature appearance, appropriate techniques and relatively clear style. On the surface, Li Sixun, Li Zhaodao and Wang Wei represent their own sovereign. However, from talking about the length of South Sect, its innovative techniques and affirming Su Shi's praise of Wang Wei, Dong Qichang is more 
respectful of the South Sect of Wang Wei.

Style Comparison of North and South Sect. Landscape painting division theory began in the Ming Dynasty. There is no clear distinction between styles in the Tang Dynasty landscape. However, since the Ming dynasty it was divided into two groups, so the two factions should have a clear distinction in style. "Tang Dynasty Famous Paintings" has a high rating on Wang Wei and Li Sixun from the theme and style of the distinction. There are also records in the "Famous Paintings of Various Dynasties" from the description of the two paintings by the Tang Dynasty and both of them are very effective in writing. The difference is that Li Sixun is good at painting trees and rocks, which scene is very wide and good at creating a far-reaching artistic conception. Meanwhile, Wang Wei's stone is similar to Wu Daozi style and the landscape is very dynamic with strange shape. They are different from the mood and style.

In the Song Dynasty, "Xuan He Painting" recorded that the Chengdu painter Li Sheng studied at Li Sixun in the late Tang Dynasty. However, his paintings were even more elegant and imaginative. At that time, some people saw his paintings and often thought they were Wang Wei's painting. Thus it can be seen that the paintings of Li Sixun and Wang Wei are also largely similar.

Xie Zhaozhe who lived in the Ming Dynasty talked about the characteristics of the landscape fine neat in "Wu ZaZu", taking Li and Wang for example and mentioning their paintings are both fine into miniature. Landscape paintings have fine features founded by Li Sixun and Li Zhaodao in the early Tang Dynasty, which let most landscape painters follow and pursuit in the future. Besides, Wang Wei's paintings also have these characteristics.

In other words, although Wang Wei was the ancestor of the South Sect and he represented a new form of ink painting. However, in fact, there is a large degree of similarity between the North Sect and South Sect in the overall style of painting, which is not an absolute distinction.

\section{Enlightenment of Research on Wang Wei's Painting under the Vision of Tang Dynasty to Teaching.}

In the teaching of Wang Wei's painting, we can draw the following conclusions from the themes of Wang Wei's painting, his contribution to ink painting and his painting style comparison with North Sect based on the perspective of the Tang Dynasty people. Wang Wei's paintings are mainly portrayals of figures in terms of quantity, but they are more diverse and representative of his landscapes. Wang Wei contributed PoMo technique to the ink painting, but the ink painting is not invented by Wang Wei. There are many ink painters in the contemporary era and there is no record of any data related to Wang Wei about the texturing methods. As an ancestor of the South Sect, Wang Wei is very similar to North Sect Li Sixun's style of painting in the Tang Dynasty. Besides, Wang Wei also drew green landscapes, which has difference compared to the general impression of Wang Wei's painting, but it is more closer to reality.

Therefore,in art history teaching, the analysis of the returned works to the times they lived in art history ,can give a relatively true impression about the works and the authors. In the long history of art history, later generations of theorists make different interpretations of the art of the past for a variety of reasons. Over time, the true image of the work has blurred or even gone far, which loses our original intention of enjoying the works of art and our desire to get acquainted with the author. Therefore, when appreciating and interpreting works of art, we should collect the documents and materials that are close to the author's time to separate the real work from the curing evaluation and cognition of later generations so as to obtain a fuller and more realistic understanding of the works and the authors.

\section{Acknowledgements}

Fund Project: This article is one of the project achievements of the general project "Two-Way Construction of Wang Wei's Poetry and Chang'an Culture" (No. 15YJA751007) of humanities and social sciences of the Chinese Ministry of Education and the special scientific research project of Shaanxi Provincial Department of Education on "Research on Wang Wei under the Vision of 
Chang'an Culture" ( No. 15JK2143).

\section{Reference}

[1] (Song) Anonymous. Xuanhe Painting Book [M]. Hunan: Hunan Fine Arts Press, 1999.

[2] (Tang) Y. Y. Zhang. Famous Paintings in Mind [M]. Shanghai: Shanghai People's Fine Arts Press, 1964.

[3] S. X. Chen. Painting History of Sui and Tang Dynasty [M]. Beijing: People's Fine Arts Press, 2001.

[4] (Qing) D. Q. Peng and others. All Poetry in Tang Dynasty [M]. Jilin: Yanbian People's Press, 2004.

[5] S. X. Chen. Chinese Painting Dynastic History Sui and Tang Paintings [M]. Beijing: People's Fine Arts Press, 2004.

[6] B. Wei. Textual Research on the Tang Dynasty [M]. Tianjin: Tianjin People's Fine Arts Press, 2007.

[7] (Tang) Wang Wei (Qing) Zhao Diancheng note. Wang Youcheng note [M]. Shanghai: Shanghai Ancient Books Press, 1998.

[8] S. J. Deng and others. Wang Wei, the Complete Works of the Top Ten Poets in Ancient China [M]. Dalian: Dalian Press, 1998.

[9] (Qing) J. Z. Zheng. Fantasy Painting Learn Concise [M]. Zhejiang: Zhejiang People's Fine Arts Press, 2017.

[10](Mei) J. H. Gao. Illustrated the History of Chinese Painting [M]. Beijing: Life. Reading. Synopsis Joint Publishing, 2014. 